\title{
A paralisia cerebral e o uso materiais recicláveis para o estímulo do desenvolvimento motor de crianças: uma revisão da literatura
}

\author{
Cerebral palsy and the use of recyclable materials to stimulate children's motor development: a
}

literature review

La parálisis cerebral y el uso de materiales reciclables para estimular el desarrollo motor de los niños: una revisión de la literatura

\section{Resumo}

No Brasil, cerca de 2,4\% das crianças com idades entre 0 e 14 anos possuem algum tipo de deficiência. A Paralisia Cerebral (PC) é uma deficiência física de caráter permanente com impactos em diversos órgãos e sistemas e, por isso, estratégias que possibilitem o pleno desenvolvimento de crianças com PC devem ser planejadas e executadas. Objetivo: Revisar as principais características da PC e abordar como a utilização de intervenções como jogos e brincadeiras utilizando materiais de baixo custo e fácil acesso pode ser útil para estimular o desenvolvimento motor de crianças com PC. Metodologia: Revisão narrativa da literatura com consulta às bases de dados PubMed e SciELO, livros e documentos de organizações, sem restrição quanto ao ano de publicação, em português e inglês. Resultados e discussão: As alterações no desenvolvimento motor de crianças com PC abrangem o movimento, equilíbrio, força e postura. As intervenções devem ocorrer o mais precocemente possíveis, em diferentes contextos, para que o crescimento e desenvolvimento seja facilitado, proporcionando melhor prognóstico. Dentre as possibilidades, a utilização de materiais de baixo custo, recicláveis e reutilizáveis é um uma estratégia que além dos benefícios motores traz mais ludicidade, prazer e dinamismo, favorecendo maior adesão às atividades. Conclusão: $\mathrm{O}$ uso de materiais de baixo custo e fácil acesso pode contribuir para a redução de déficits funcionais e desenvolvimento motor de crianças com PC. A construção de jogos e brincadeiras com esses materiais além deixar as intervenções mais atrativas pode ser aplicada em diferentes ambientes, incluindo o domiciliar e o escolar.

Palavras-chave: Pessoas com deficiência; Transtornos motores; Jogos e brinquedos; Desenvolvimento sustentável.

\begin{abstract}
In Brazil, about $2.4 \%$ of children aged between 0 and 14 years have some type of disability. Cerebral Palsy (CP) is a permanent physical disability with impacts on various organs and systems and, therefore, strategies that enable the full development of children with $\mathrm{CP}$ must be planned and implemented. Objective: To review the main characteristics of CP and discuss how the use of interventions such as games and games using inexpensive and easily accessible materials can be useful to stimulate the motor development of children with CP. Methodology: Narrative literature review by consulting the PubMed and SciELO databases, books and documents from organizations, without restriction as to the year of publication, in Portuguese and English. Results and discussion: Changes in the motor development of children with CP include movement, balance, strength and posture. Interventions should occur as early as possible, in different contexts, so that growth and development is facilitated, providing a better prognosis. Among the possibilities, the use of low-cost, recyclable and reusable materials is a strategy that, in addition to motor benefits, brings more playfulness, pleasure and dynamism, favoring greater adherence to activities. Conclusion: The use of inexpensive and easily accessible materials can contribute to the reduction of functional deficits and motor development in children with CP. The construction of games and games with these materials, in addition to making interventions more attractive, can be applied in different environments, including the home and school.
\end{abstract}

Keywords: Disabled persons; Motor disorders; Play and playthings; Sustainable development. 


\begin{abstract}
Resumen
En Brasil, alrededor del 2,4\% de los niños de 0 a 14 años tienen algún tipo de discapacidad. La Parálisis Cerebral (PC) es una discapacidad física permanente con impactos en diversos órganos y sistemas, por lo que se deben planificar e implementar estrategias que permitan el pleno desarrollo de los niños con PC. Objetivo: Revisar las principales características de la PC y discutir cómo el uso de intervenciones como juegos y juegos con materiales económicos y de fácil acceso puede ser útil para estimular el desarrollo motor de los niños con PC. Metodología: Revisión de literatura narrativa mediante la consulta de bases de datos PubMed y SciELO, libros y documentos de organizaciones, sin restricción en cuanto al año de publicación, en portugués e inglés. Resultados y discusión: Los cambios en el desarrollo motor de los niños con parálisis cerebral incluyen el movimiento, el equilibrio, la fuerza y la postura. Las intervenciones deben ocurrir lo antes posible, en diferentes contextos, para que se facilite el crecimiento y el desarrollo, proporcionando un mejor pronóstico. Entre las posibilidades, el uso de materiales de bajo costo, reciclables y reutilizables es una estrategia que, además de los beneficios motores, aporta más alegría, placer y dinamismo, favoreciendo una mayor adherencia a las actividades. Conclusión: El uso de materiales económicos y de fácil acceso puede contribuir a la reducción de los déficits funcionales y del desarrollo motor en niños con parálisis cerebral. La construcción de juegos y juegos con estos materiales, además de hacer más atractivas las intervenciones, se puede aplicar en diferentes entornos, incluido el hogar y la escuela.
\end{abstract}

Palabras clave: Personas con discapacidad; Trastornos motores; Juego e implementos de juego; Desarrollo sostenible.

\title{
1. Introdução
}

Segundo a Lei Brasileira de Inclusão da Pessoa com Deficiência, a pessoa com deficiência é definida como "aquela que tem impedimento de longo prazo de natureza física, mental, intelectual ou sensorial, o qual, em interação com uma ou mais barreiras, pode obstruir sua participação plena e efetiva na sociedade em igualdade de condições com as demais pessoas" (Presidência da República, 2015, on-line). A Organização Mundial da Saúde (OMS) estima que em todo o mundo mais de um bilhão de pessoas possuam algum tipo de deficiência (World Health Organization, 2021). No Brasil, o Censo Demográfico de 2010 (Instituto Brasileiro de Geografia e Estatística, 2012), último até a presente data sobre deficiência, mostrou que aproximadamente $24 \%$ da população possui algum tipo de deficiência, e desse total, 6,7\% com formas mais severas, ou seja, em torno de 13 milhões de brasileiros.

A Paralisia Cerebral (PC), também denominada de Encefalopatia Crônica Não Progressiva, é a deficiência física mais comum da infância, com incidência estimada entre 1,5 e 3/ 1000 nascidos-vivos (Sadowska, et al., 2020). Ocorre devido à uma lesão no cérebro, com repercussões em aspectos que incluem o equilíbrio postural, o movimento, a coordenação motora e a postura, e que variam de acordo com o local e extensão da área da lesão (Vitrikas, et al., 2020). Crianças com PC também tendem a apresentar capacidade cardiorrespiratória reduzida quando comparadas com crianças sem PC (Verschuren, et al., 2016), assim como menor força muscular (Merino-Andrés, et al., 2021) e menor participação programas de atividades físicas (Wright, et al., 2019). Esses fatores contribuem para o aumento do risco de doenças cardiovasculares e pior estado geral de saúde (Ryan, et al., 2019), e dessa forma, o estímulo à prática de atividades e exercícios físicos deve ser constante e começar precocemente.

Em se tratando de crianças, as ofertas de atividades devem ser amplas, atrativas e lúdicas, de modo a atrair a atenção e facilitar a adesão. Não devem ser restritas e exclusivas aos ambientes de reabilitação, fazendo parte da rotina da criança em diferentes contextos incluindo a escola (Dantas, et al., 2020) e o domicílio. Para tal, a adaptação dos implementos, do espaço e das atividades frequentemente se faz necessária, variando em função das características individuais. A realização de tais adaptações, no entanto, não exige obrigatoriamente o uso de materiais de alto custo financeiro, sofisticação e tecnologia; pelo contrário, materiais simples, de fácil acesso e de baixo custo, como é o caso dos materiais recicláveis e reutilizáveis, podem ser utilizados conforme demonstrado recentemente (Vigário, et al., 2021a; Vigário, et al., 2021b; Vigário, et al., 2021c; Vigário, et al., 2021d) e pode envolver a participação da própria criança no processo.

Extrapolando para outros cenários, o envolvimento da criança na construção e adaptação de jogos, brinquedos e implementos também traz como vantagens o estímulo à criatividade e à consciência ambiental por meio do reaproveitamento 
de materiais, conferindo a multidisciplinaridade no processo. Não se pode deixar de destacar que a Agenda 2030 da Organização das Nações Unidas (ONU) prevê que para que o mundo seja sustentável para a atual e as futuras gerações é necessária redução da geração de resíduos por meio da reciclagem e reuso (Plataforma Agenda 2030, 2015).

Uma vez que as adaptações e a construção de implementos, brinquedos e jogos podem ser feitos utilizando materiais de baixo custo, é essencial que estas informações cheguem ao alcance da população que lida com crianças com PC, incluindo profissionais, estudantes de graduação (formação e estágio) e familiares. Assim, contribui-se para que esta prática mais difundida e adotada em centros de reabilitação, escolas e por familiares no ambiente domiciliar. Nessa perspectiva, o presente estudo de revisão narrativa da literatura teve como objetivo reunir informações sobre as principais características da PC, e abordar como o uso de materiais de baixo custo e fácil acesso, como é o caso dos materiais recicláveis e reutilizáveis, pode auxiliar no desenvolvimento motor de crianças com PC.

\section{Metodologia}

Foi realizada uma revisão narrativa da literatura com consulta às bases de dados PubMed e SciELO, livros, documentação técnica, normas, decretos e portarias. Foram considerados os textos publicados nos idiomas português e inglês e sem restrição quanto ao ano de publicação. Os seguintes descritores foram utilizados para a busca do material bibliográfico com seus respectivos termos correspondentes em inglês: paralisia cerebral, pessoa com deficiência, desenvolvimento motor, transtorno motor, funcionalidade, jogos, brincadeiras e reabilitação. Os termos foram usados de forma isolada e combinada.

\section{Resultados e Discussão}

\subsection{Paralisia cerebral: conceitos, características e aspectos epidemiológicos}

A PC é definida como um grupo de desordens de caráter permanente do desenvolvimento da postura e do movimento, que causam limitações em atividades do cotidiano. Caracteriza-se por um distúrbio não progressivo que ocorre durante o desenvolvimento encefálico durante as fases pré-natal, perinatal ou pós-natal, com maior incidência nas duas primeiras - em especial nos países em desenvolvimento (Gulati \& Sondhi, 2018). Dentre as principais dificuldades de desenvolvimento motor se destacam as limitações de movimentos articulares, devido a contraturas de membros inferiores (Herskind, et al., 2016). Barber \& Boyd (2016) reforçam que crianças com PC quando comparadas às crianças sem deficiência possuem menor crescimento do músculo gastrocnêmio, fato este que afeta diretamente o controle de marcha, o equilíbrio postural, a geração de força e o suporte de peso.

Embora a identificação dos sinais marcadores da PC fiquem mais evidentes entre os 3 e 5 anos de idade, alguns podem sugerir uma investigação antecipada, a destacar: (i) neurocomportamentais: letargia, pouca atenção visual, irritabilidade excessiva e distúrbios do sono; (ii) desenvolvimento: atraso ou exagero em reflexo de desenvolvimento, dificuldade de sentar e persistente extensão de pernas; (iii) tônus e postura motoras: aumentado ou diminuído, assim como o controle inadequado de cabeça e padrões anormais de motricidade e (iv) principais marcos motores: o atraso em sentar, engatinhar, controle de tronco e andar (Blascovi-assis, 2015).

Estima-se que a incidência de PC seja de 2 a 3 bebês entre 1000 nascidos-vivos (Vitrikas, et al., 2020), sendo maior em países em desenvolvimento, com 7 casos entre 1000 nascidos-vivos (Baltor \& Dupas, 2013). Esta diferença se relaciona à qualidade dos serviços de saúde no atendimento primário às gestantes e aos cuidados durante o pré-natal. No Brasil, existe uma deficiência de estudos que tenham investigado a prevalência e a incidência de PC (Brasil, 2013) e, por isso, considera-se o número de casos de países em desenvolvimento. Recentemente, um estudo epidemiológico conduzido em Aracajú/ Sergipe, Brasil revelou que a prevalência de período de PC na capital era de 1,37 em cada 1000 habitantes, sendo a maior parte homem 
de raça/ cor parda ou preta, e que a família vive em situação de vulnerabilidade (Peixoto, et al., 2020). Em São Paulo, dos 743 pacientes que deram início ao acompanhamento na Associação de Assistência à Criança Deficiente (AACD) entre os anos de 2012 e 2014, a maior parte também era do sexo masculino (52,6\%), pré-termo ao nascimento (50,7\%) com parto cesáreo (56,5\%) e diparético espástico (33,9\%) em relação ao tipo de PC (Binha, et al., 2018). No Ceará, de um grupo de 122 crianças com PC em atendimento ambulatorial, $72 \%$ apresentavam PC espástica, a anóxia neonatal era a principal causa para a maioria (52,5\%) e o diagnóstico ocorreu até o primeiro mês de vida na menor parte do grupo (38,5\%) (Cavalcante, et al., 2017).

O diagnóstico da PC é feito primariamente com base em sinais e sintomas clínicos. Secundariamente, o exame de ressonância magnética pode ser utilizado para confirmar a existência de uma lesão cerebral, sobretudo em casos quando não há um motivo claro que justifique os sinais e sintomas apresentados pelo paciente (Vitrikas, et al., 2020). A avaliação clínica inclui a aplicação de escalas como a General Movements Assessment ou Hammersmith Infant Neurological Examination em crianças de até cinco meses de idade, e em crianças com mais de cinco meses de idade escalas como a Hammersmith Infant Neurological Examination ou Developmental Assessment of Young Children, todas conduzidas por profissionais qualificados e especializados (Novak, et al., 2017).

Em relação às classificações, a PC pode ser (i) espástica, (ii) discinética, (iii) atáxica, (iv) mista e (v) hipotônica (Novak, et al., 2017; Vitrikas, et al., 2020). As principais características clínicas relacionadas a cada classificação da PC estão descritas a seguir.

(i) Espástica: A espasticidade tem como principal característica o aumento do tônus muscular com intensificação dos reflexos profundos, decorrente de hiperexcitabilidade do reflexo do estiramento (Teive, et al., 1998). Possui predominância nos músculos flexores dos membros superiores e extensores dos membros inferiores. Geralmente, a criança com PC espástica possui grande dificuldade de estender o corpo, permanecendo com os membros superiores, tronco, quadris e joelhos semiflexionados durante a maior parte do tempo (Souza, et al., 2014). Com base no local de comprometimento, a PC espástica se divide em: (a) tetra ou quadriparética - comprometimento dos quatro membros, com lesão encefálica bilateral, extensa, podendo ser simétrica ou não, sendo a forma mais grave da PC espástica. São os pacientes com maior grau de comprometimento, sendo totalmente dependentes para execução das atividades da vida diária. A deficiência intelectual é bastante frequente, com variação de intensidade; (b) bi ou diparética - predominância nos membros inferiores, sendo mais recorrente em prematuros. Os membros superiores também costumam ser acometidos, porém em intensidades menores e variadas. Raramente conseguem realizar marcha independente antes de 2 anos, sendo um sinalizador importante na avaliação; e (c) hemiparética - o comprometimento acontece em apenas um dos lados, sendo de fácil percepção, ainda nos primeiros 12 meses (Embiruçu, et al., 2015). Em 75\% dos casos, a origem acontece por problemas na fase pré-natal. Em mais da metade dos casos, surgem o comprometimento cognitivo e distúrbios de aprendizagem, assim como associação com epilepsia. A PC espástica é a mais comum entre as PC, com percentuais variando entre 70 e 91\% dos casos (Souza, et al., 2014).

(ii) Discinética: Caracteriza-se pela presença de movimentos involuntários que se sobrepõem aos movimentos voluntários (Embiruçu, et al., 2015), posturas inadequadas associadas à falta de coordenação motora, resultantes da ativação simultânea dos músculos agonistas e antagonistas, gerando alteração na regulação do tônus muscular. Representa cerca de 8 a 15\% dos casos de PC. Os sinais e sintomas da PC discinética são percebidos em crianças próximo aos 12 meses de idade, iniciando com a hipotonia muscular que evolui, em seguida, para hipertonia, a partir do aumento do tônus muscular. É mais evidente nas extremidades ou na realização de movimentos mais suaves e, durante o sono, normalmente estes sinais desaparecem. A PC discinética pode ser apresentada nas seguintes subdivisões: (a) coreoatetóide, também chamada de hipercinética. Os indivíduos apresentam boa amplitude de movimento das articulações, embora não consigam controlar os atos motores; apresentam movimentos abruptos, sem direção e desorganizados, quando realizam movimento voluntário. As funções 
cognitivas costumam ser preservadas, mas dificuldades na fala são frequentes; (b) distônica, normalmente identificável no segundo ano de vida da criança. Costuma ser menos frequente que a PC coreoatetóide, sendo também chamada de hipocinesia. Os indivíduos possuem desordem motora com aumento súbito do tônus muscular (hipertonia) durante a realização de movimentos voluntários, mudanças de postura ou alguma reação emocional (Gulati \& Sondhi, 2017).

(iii) Atáxica: $\mathrm{Na}$ maioria dos casos, possui origem pré-natal com comprometimento do cerebelo, que coordena o equilíbrio e funções de coordenação. Também pode ter origem genética como a Síndrome de Joubert ou Síndrome de DandyWalker (Fonseca, et al. 2011; Embiriçu, et al., 2009). Alterações na fala são muito frequentes, assim como o atraso no desenvolvimento. Nos meses iniciais de vida, o bebê pode apresentar hipotonia, retardando as aquisições motoras visivelmente para mudança de decúbito, assim como movimentos oscilatórios e instáveis de cabeça e tronco. Ainda no primeiro ano de vida, percebe-se a dificuldade em alcançar objetos, dismetria e falta de coordenação motora. As crianças raramente conseguem deambular de forma independente antes dos quatro anos de idade, podem ter alterações cognitivas, porém de forma leve e possuem grande dificuldade na motricidade fina (Embiruçu, et al., 2015; Tarran, et al., 2015).

(iv) Mista: Conforme o próprio nome sugere, a PC Mista representa a combinação dos sinais e sintomas dos demais tipos de PC e sua prevalência varia de 9 a 22\% dos casos (Tarran, et al., 2015). Deve-se sempre citar as características da paralisia predominante, acompanhado do nome da que a acompanha, para facilitar o entendimento. Embiruçu et al. (2015) afirmam que as combinações dos sintomas podem ser de dois ou mais tipos de PC.

(v) Hipotônica: Mais rara, possui prevalência estimada de aproximadamente 1\% dos casos de PC (Gauzzi \& Fonseca, 2004). Por este fato, alguns autores não a citam como forma de classificação, gerando controvérsia entre os pesquisadores. Tem como característica principal a hipotonia persistente, que ultrapassa os dois anos de vida, não sendo resultante de lesão primária muscular ou de neurônios periféricos. Por ter fisiopatologia e etiologia heterogêneas, acaba gerando dificuldade numa compreensão mais clara e eficaz, acreditando-se que tenha origem a partir de lesão nos mecanismos reguladores centrais ou ainda, lesão celular na parte anterior da medula. Reforçam Embiruçu et al. (2015), que os pacientes possuem dificuldade em manter a postura corporal, com rara possibilidade de desenvolver marcha independente, devido à hipotonia que o acompanha ao longo da vida.

A avaliação dos déficits e do nível de comprometimento no desenvolvimento motor de crianças e adolescentes com PC é feita comumente por meio do uso de escalas. Segundo Mélo (2011) as escalas são importantes ferramentas clínicas para profissionais da área de reabilitação. Visam auxiliar e mensurar a percepção do desenvolvimento infantil, a destacar: a postura corporal, o tônus muscular, as reações posturais, os movimentos espontâneos, a coordenação motora, o reflexo primitivo, dentre outros. Algumas escalas de classificação da avaliação do desenvolvimento motor incluem: (i) Medida da Função Motora Grossa [GMFM], desenvolvida em 1990 e revisada em 1993 para avaliar quantitativamente os aspectos motores e dinâmicos especificamente de crianças com PC; (ii) Avaliação Pediátrica do Inventário de Incapacidades [PEDI], desenvolvida em 1992 e revalidada em 2010 (Haley, et al., 2010); (iii) Alberta Motor Infant Scale [AIMS], desenvolvida pelo Departamento de Medicina e Reabilitação da Universidade de Alberta no Canadá (1994); (iv) Sistema de Classificação da Função Motora Grossa [GMFCS], criado em 1997 utilizando parâmetros do GMFM. Utiliza de subdivisões por faixas etárias, focando as habilidades motoras de membros inferiores (Damiano, et al., 2006). A escala original GMFM é composta por 88 itens, que a fim de aperfeiçoamento de protocolo foram reduzidos para 66 itens em 2000 (GMFM-66); (v) General Movements Assessment (Bosanquet, et al., 2013) e (vi) Hammersmith Infant Neurological Examination (Romeo, et al., 2016). 


\subsection{O uso de materiais recicláveis como ferramenta facilitadora para o desenvolvimento motor de crianças com paralisia cerebral.}

As manifestações físico-motoras descritas na PC incluem alteração no equilíbrio postural e coordenação motora, diminuição da força e do tônus muscular e perda do controle motor, impactando diretamente na postura e no movimento (Vitrikas, et al., 2020). A funcionalidade comprometida limita a participação e a realização das atividades de vida diária, diminui as oportunidades e favorece a exclusão social. Por isso, estímulos físico-motores devem acompanhar a vida da criança com PC em diferentes contextos, tanto em programas de reabilitação, quanto em escolas e no ambiente em que vive.

Os estímulos devem ser voltados não somente para os marcos motores do desenvolvimento, isto é, controlar a cabeça, rolar, arrastar, sentar, engatinhar e marchar, como também para as atividades de vida diária, como se vestir, locomover, alimentar, tomar banho, escovar os dentes, entre outros (Monteiro, et al., 2010). Estes estímulos devem acontecer de forma precoce e contínua, a fim de amenizar as repercussões físicas e possíveis problemas musculoesqueléticos secundários.

Um estudo de revisão sistemática mostrou que intervenções como treinamento bimanual, terapia por contensão induzida, treinamento específico de tarefa e enriquecimento do ambiente (espaço físico) onde a criança vive com equipamentos, materiais, brinquedos e jogos que possam estimular a curiosidade e a realização de movimentos de modo independente causam efeitos positivos, associadas à melhor saúde e desenvolvimento motor e cognitivo (Novak, et al., 2020).

A terapia por contensão induzida, por exemplo, se relaciona ao treinamento funcional dos membros superiores de em quadros de hemiplegia, muito frequente na PC. Crianças com hemiplegia tendem a priorizar o membro menos afetado como estratégia para facilitar a realização das tarefas diárias, levando a um "desuso aprendido", isto é, a diminuição do uso do hemisfério afetado (Anjos, et al., 2016). O protocolo da terapia por contensão induzida envolve: o treinamento intensivo direcionado a tarefa, métodos de transferência e restrição do membro menos afetado (geralmente o uso de talas de gesso) e métodos de adesão de modo a transferir os ganhos obtidos em ambiente clínico para o ambiente real do paciente (Fonseca \& Guarany, 2018). Embora seja evidente que para algumas dessas intervenções sejam necessários materiais e equipamentos específicos para a sua aplicação, assim como profissionais especializados, recursos acessíveis tais como materiais recicláveis e reutilizáveis podem ser utilizados para esta finalidade. Como vantagem, adiciona-se o fato de serem materiais presentes no ambiente domiciliar, e por isso, a possibilidade do estímulo ser continuado fora do ambiente clínico. Com uma diversidade de materiais, incluindo por exemplo, latas de alumínio, caixas de papelão, tampas de garrafas e copos plásticos podem trabalhados movimentos de preensão, encaixe, cruzamento da linha média, força, extensão e flexão de cotovelos, que frequentemente estão comprometidos em crianças com PC (Fonseca \& Guarany, 2018). Em cenários de baixa renda e acesso limitado à recursos, a utilização desses materiais possui uma relevância ainda maior (Bramante \& Sparti, 2021).

Nos contextos motivacional e de criatividade, a utilização de materiais recicláveis e reutilizáveis pode ser uma estratégia que favoreça não somente o desenvolvimento motor, como também a ludicidade em todo o processo, uma vez que a própria criança pode participar da construção dos objetos, além de brinquedos e jogos. Peres et al. (2018) demonstraram por meio de um estudo de revisão integrativa com 20 estudos que o uso da ludicidade tendo como objetivo o aprimoramento da motricidade fina, da motricidade grossa, do equilíbrio e da marcha é efetivo em crianças com PC. Além dos benefícios diretos nas habilidades motoras, torna o tratamento mais prazeroso e dinâmico. O ato de brincar - com objetos, com outras crianças/ pessoas e com o próprio corpo - contribui para o desenvolvimento motor, emocional, cognitivo e sensorial, e autoconhecimento da criança atraso no desenvolvimento neuropsicomotor (Brasil, 2016).

Recentemente, Vigário et al. (2021a, 2021b, 2021c e 2021d) e Ribeiro (2021) demonstraram a construção de uma série de materiais pedagógicos, brinquedos e implementos para a prática de esportes por crianças com deficiência a partir de materiais recicláveis, conforme exemplo na Figura 1. Observa-se que o processo não envolve grande complexidade, é simples, 
e pode ser realizado por crianças com auxílio de adultos, ou dependendo do nível de independência, pela própria criança. Nesse cenário, além do estímulo ao desenvolvimento motor, pode-se ainda trabalhar outras questões importantes para a formação da criança, tais como a educação em meio ambiente e a promoção do desenvolvimento sustentável. Conceitos como o descarte adequado e redução da geração do lixo e reciclagem, presentes na Agenda 2030 da ONU para o desenvolvimento sustentável, podem ser introduzidos de uma forma lúdica contribuindo para uma maior conscientização ambiental.

Figura 1 - Etapas para a criação de uma bola a partir de garrafa PET, fita adesiva e jornal.

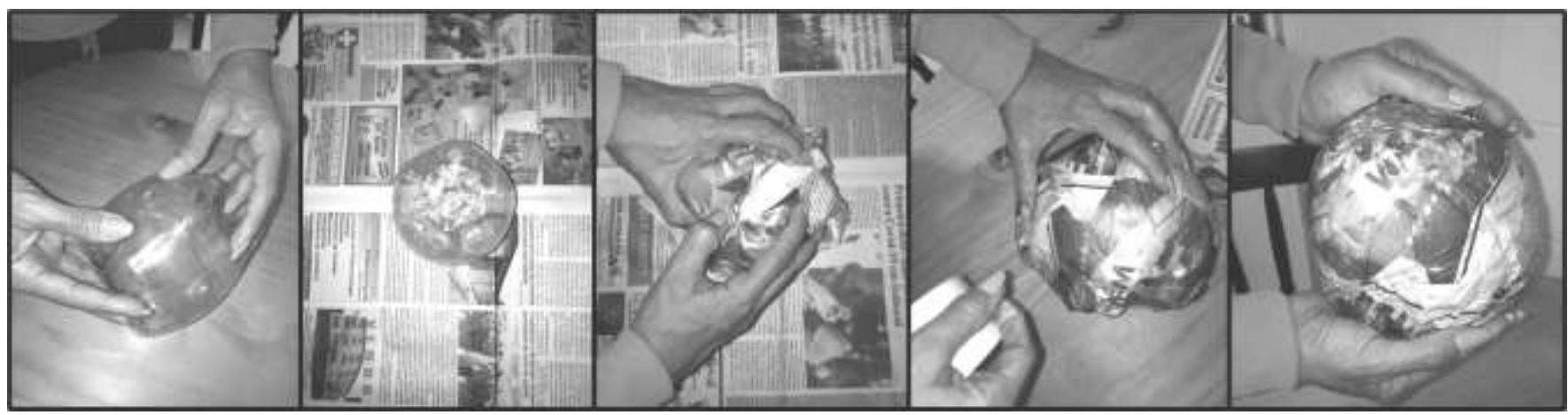

Fonte: Adaptado de Vigário et al. (2021d) com autorização dos autores.

\section{Conclusão}

Crianças com PC apresentam alterações no desenvolvimento motor com repercussões no movimento, equilíbrio e postura, com variações manifestações clínicas de acordo com a classificação da PC. As intervenções para minimizar tais repercussões devem ocorrer de forma precoce, em diferentes contextos, de modo a favorecer o melhor desenvolvimento da criança. A utilização de materiais de baixo custo, como é o caso de materiais recicláveis e reutilizáveis, é uma estratégia que pode contribuir para o desenvolvimento motor das crianças com PC tanto por meio da reprodução/ adaptação de implementos que são tradicionalmente utilizados na reabilitação, quanto pela possibilidade de criação de jogos e brinquedos, também importantes neste processo, conferindo uma maior ludicidade, prazer e dinamismo. Além disso, devido à facilidade de acesso a estes materiais, os estímulos para o desenvolvimento motor podem ser continuados e desenvolvidos também no ambiente domiciliar. Dentro de uma perspectiva socioeconômica, a utilização desses materiais é particularmente importante em populações de baixa renda e que possuem limitado acesso à recursos terapêuticos e, por isso, esforços devem ser feitos para que tais informações sejam difundidas. Por fim, o aspecto ambiental não pode deixar de ser considerado, uma vez que conceitos como sustentabilidade, preservação ao meio ambiente e desenvolvimento sustentável podem ser trabalhados.

\section{Agradecimentos}

Esse estudo foi parcialmente financiado pela Coordenação de Aperfeiçoamento de Pessoal de Nível Superior - Brasil (CAPES) - Código de financiamento 001, pela Fundação de Amparo à Pesquisa do Estado do Rio de Janeiro (FAPERJ) (edital E-26/203.256/2017) e pelo Conselho Nacional de Desenvolvimento Científico e Tecnológico (CNPq).

\section{Referências}

Anjos, E. S., Pacheco, F. Y. R. \& Santos, R. C. C. S. (2016). Terapia de Contensão Induzida na função do membro superior parético. Revista da Sociedade Brasileira de Clínica Médica, 14(3), 172-176.

Baltor, M. R., \& Dupas, G. (2013). Experiences from families of children with cerebral paralysis in context of social vulnerability. Revista Latino-americana de Enfermagem, 21(4), 956-963. https://doi.org/10.1590/S0104-11692013000400018 
Brasil. (2013). Ministério da Saúde. Secretaria de Atenção à Saúde. Departamento de Ações Programáticas Estratégicas. Diretrizes de atenção à pessoa com paralisia cerebral. Brasília. 80 p. : il

Brasil. (2016). Ministério da Saúde. Secretaria de Atenção à Saúde. Diretrizes de estimulação precoce: crianças de zero a 3 anos com atraso no desenvolvimento neuropsicomotor / Ministério da Saúde, Secretaria de Atenção à Saúde. - Brasília: Ministério da Saúde, 184 p. : il.

Barber, L. A., \& Boyd, R. N. (2016). Growing muscles in children with cerebral palsy. Developmental Medicine and Child Neurology, 58(5), 431-432. https://doi.org/10.1111/dmcn.12980

Binha, A. M. P., Maciel, S. C., \& Bezerra, C. C. A. (2018) Perfil epidemiológico dos pacientes com paralisia cerebral atendidos na AACD - São Paulo. Acta Fisiátrica, 25(1), 1-6. https://doi.org/10.11606/issn.2317-0190.v25i1a158818

Blascovi-assis, S. M. (2015). Desenvolvimento motor e suas alterações na paralisia cerebral. In: Monteiro, C. B. M., Abreu, L.C. \& Valenti, V. E. (Orgs). Paralisia cerebral: teoria e prática. São Paulo: Plêiade.

Bosanquet, M., Copeland, L., Ware, R., \& Boyd, R. (2013). A systematic review of tests to predict cerebral palsy in young children. Developmental Medicine and Child Neurology, 55(5), 418-426. https://doi.org/10.1111/dmcn.12140

Bramante, A. C., Sparti, S. C. M. (2021). O desenvolvimento motor do pré-escolar e à utilização de material reciclável. Revista de Estudos Universitários $R E U$, [S. l.], 8 (1), 95-139. Disponível em: http://periodicos.uniso.br/ojs/index.php/reu/article/view/4488. Acesso em: 5 nov. 2021.

Cavalcante, V., Martins, M., Oriá, M., Ximenes, L., Frota, M., \& Carvalho, Z. (2017). Perfil epidemiológico das crianças com paralisia cerebral em atendimento ambulatorial [Epidemiological profile of children with cerebral palsy receiving care at outpatient clinics]. Revista Enfermagem UERJ, 25, e8780. doi:https://doi.org/10.12957/reuerj.2017.8780

Damiano, D., Abel, M., Romness, M., Oeffinger, D., Tylkowski, C., Gorton, G., Bagley, A., Nicholson, D., Barnes, D., Calmes, J., Kryscio, R., \& Rogers, S. (2006). Comparing functional profiles of children with hemiplegic and diplegic cerebral palsy in GMFCS Levels I and II: Are separate classifications needed?. Developmental Medicine and Child Neurology, 48(10), 797-803. https://doi.org/10.1017/S0012162206001733

Dantas, M. J. B., Dantas, T. L. F. S., \& Gorla, J. I. (2020). Physical education in the context of the person with cerebral palsy: profile of boccia teachers. Research, Society and Development, 9(7), e432974347. https://doi.org/10.33448/rsd-v9i7.4347

Embiruçu, E. K., Martyn, M. L., Schlesinger, D., \& Kok, F. (2009). Autosomal recessive ataxias: 20 types, and counting. Arquivos de Neuro-psiquiatria, 67(4), 1143-1156. https://doi.org/10.1590/s0004-282x2009000600036

Embiruçu, E. K., Monteiro, C. B. M., Silva, T. D., Reis, A. O. A., Valenti, V. E., Oliveira, A. G. \& Abreu, L.C. (2015). Paralisia cerebral. In: Monteiro, C. B. M., Abreu, L.C. \& Valenti, V. E. (Orgs). Paralisia cerebral: teoria e prática. São Paulo: Plêiade.

Fonseca, L. F., Xavier, C.C., \& Pianetti, G. (2011). Compêndio de neurologia infantil. 2. ed. Rio de Janeiro: MedBook

Fonseca, C. S. \& Guarany, N. R. (2018). A intervenção por terapia de contensão induzida no desempenho. Revista Interinstitucional Brasileira de Terapia Ocupacional, 2(2), 292-304.

Gauzzi, L. D. V. \& Fonseca, L. F. (2004). Classificação da paralisia cerebral. In: Lima, C. L. F. A. \& Fonseca, L. F. Paralisia Cerebral: neurologia, ortopedia e reabilitação. Rio de Janeiro: Guanabara Koogan.

Gulati, S., \& Sondhi, V. (2018). Cerebral Palsy: An Overview. Indian Journal of Pediatrics, 85(11), 1006-1016. https://doi.org/10.1007/s12098-017-2475-1

Instituto Brasileiro de Geografia e Estatística (IBGE). Censo Brasileiro de 2010. Rio de Janeiro: IBGE, 2012.

Vitrikas, K., Dalton, H., \& Breish, D. (2020). Cerebral Palsy: An Overview. American Family Physician, 101(4), 213-220.

Haley, S. M., Coster, W. I., Kao, Y. C., Dumas, H. M., Fragala-Pinkham, M. A., Kramer, J. M., Ludlow, L. H., \& Moed, R. (2010). Lessons from use of the Pediatric Evaluation of Disability Inventory: where do we go from here? Pediatric physical therapy : the official publication of the Section on Pediatrics of the American Physical Therapy Association, 22(1), 69-75. https://doi.org/10.1097/PEP.0b013e3181cbfbf6

Herskind, A., Ritterband-Rosenbaum, A., Willerslev-Olsen, M., Lorentzen, J., Hanson, L., Lichtwark, G., \& Nielsen, J. B. (2016). Muscle growth is reduced in 15-month-old children with cerebral palsy. Developmental Medicine and Child Neurology, 58(5), 485-491. https://doi.org/10.1111/dmcn.12950

Mélo, T. R. (2011). Escalas de avaliação do desenvolvimento e habilidades motoras: AIMS, PEDI, GMFM e GMFCS. In: Castilho-Weinert, L. V. \& FortiBellani, C. D. (Ed) Fisioterapia em Neuropediatria. Curitiba, PR, OMNIPAX.

Merino-Andrés, J., García de Mateos-López, A., Damiano, D. L., \& Sánchez-Sierra, A. (2021). Effect of muscle strength training in children and adolescents with spastic cerebral palsy: A systematic review and meta-analysis. Clinical Rehabilitation, 2692155211040199. Advance online publication. https://doi.org/10.1177/02692155211040199

Monteiro,C. B.M., Jakabi, C. M., Palma, G. C. S., Torriani-Pasin, C., \& Meira Junior, C. M. (2010). Aprendizagem motora em crianças com paralisia cerebral. Journal of Human Growth and Development, 20(2), 250-262.

Novak, I., Morgan, C., Adde, L., Blackman, J., Boyd, R. N., Brunstrom-Hernandez, J., Cioni, G., Damiano, D., Darrah, J., Eliasson, A. C., de Vries, L. S., Einspieler, C., Fahey, M., Fehlings, D., Ferriero, D. M., Fetters, L., Fiori, S., Forssberg, H., Gordon, A. M., Greaves, S., ... Badawi, N. (2017). Early, Accurate Diagnosis and Early Intervention in Cerebral Palsy: Advances in Diagnosis and Treatment. JAMA Pediatrics, 171(9), 897-907. https://doi.org/10.1001/jamapediatrics.2017.1689 
Peixoto, M. V. da S., Duque, A. M., Carvalho, S. de, Gonçalves, T. P., Novais, A. P. de S., \& Nunes, M. A. P. (2021). Características epidemiológicas da paralisia cerebral em crianças e adolescentes em uma capital do nordeste brasileiro. Fisioterapia e Pesquisa, 27(4), 405-412. https://doi.org/10.1590/1809$2950 / 20012527042020$

Peres, L. W., Leite, A. C. A. B., Alvarenga, W. de A., Al Ghazaoui, M. M., Rahall, T. M., \& Nascimento, L. C. (2018). Estratégias lúdicas na reabilitação motora de crianças com paralisia cerebral: revisão integrativa. Revista Eletrônica de Enfermagem, 20. https://doi.org/10.5216/ree.v20.50936

Plataforma Agenda 2030. (2015). Os 17 Objetivos de Desenvolvimento Sustentável. Acesso em Novembro 1, 2021, de http://www.agenda2030.com.br/ods/12/ Presidência da República. (2015). Lei no 13146, de 6 de julho de 2015. Acesso em Novembro 1, 2021, de http://www.planalto.gov.br/ccivil_03/_ato20152018/2015/lei/113146.htm

Ribeiro, A. H. (2021). Elaboração de materiais pedagógicos e esportivos para aulas de educação física adaptada. In R. C. S. Mocarzel (Org), Deficiência e Transtornos em Educação Física (pp.155-176). Curitiba: Appris Editora.

Ryan, J. M., Peterson, M. D., Ryan, N., Smith, K. J., O'connell, N. E., Liverani, S., Anokye, N., Victor, C., \& Allen, E. (2019). Mortality due to cardiovascular disease, respiratory disease, and cancer in adults with cerebral palsy. Developmental Medicine and Child Neurology, 61(8), 924-928. https://doi.org/10.1111/dmcn.14176

Romeo, D. M., Ricci, D., Brogna, C., \& Mercuri, E. (2016). Use of the Hammersmith Infant Neurological Examination in infants with cerebral palsy: a critical review of the literature. Developmental medicine and child neurology, 58(3), 240-245. https://doi.org/10.1111/dmcn.12876

Rosenbaum, P., Paneth, N., Leviton, A., Goldstein, M., Bax, M., Damiano, D., Dan, B., \& Jacobsson, B. (2007). A report: the definition and classification of cerebral palsy April 2006. Developmental Medicine and Child Neurology. Supplement, 109, 8-14.

Sadowska, M., Sarecka-Hujar, B., \& Kopyta, I. (2020). Cerebral Palsy: Current Opinions on Definition, Epidemiology, Risk Factors, Classification and Treatment Options. Neuropsychiatric Disease and Treatment, 16, 1505-1518. https://doi.org/10.2147/NDT.S235165

Souza, A. M. C. Gondim, C. M. L. \& Van der Linden Junior, H. (2014). Paralisia cerebral: história, conceito e classificações. In: Souza, A. M . C. \& Daher, S. (Orgs). Reabilitação: paralisia cerebral. Goiânia: Cânone.

Tarran, A. B. P., Castro, N. M. D., Morais Filho, M. C. \& Abreu, F. P. (2015). Paralisia cerebral. In: Fernandes, A. C., Ramos, A. C. R., Moraes Filho, M. C.\& Ares, M. J. J, (Coord). Reabilitação. 2. ed. Barueri: Manole.

Teive, H. A. G., Zonta, M., \& Kumagai, K. (1998). Tratamento da espasticidade: uma atualização. Arquivos de Neuro-psiquiatria,56(4):852-858.

Verschuren, O., Peterson, M. D., Balemans, A. C., \& Hurvitz, E. A. (2016). Exercise and physical activity recommendations for people with cerebral palsy. Developmental Medicine and Child Neurology, 58(8), 798-808. https://doi.org/10.1111/dmcn.13053

Vigário, P., Pena, B. G., Telles, S. C. C., Souza, A. C.C., Barros, I. C. S. S., Cortat, J. M. \& Silva, G. S. (2021a). Atletismo Olímpico e Paralímpico. Esporte, Inclusão e Sustentabilidade. Rio de Janeiro: Gama Assessoria Empresarial.

Vigário, P., Pena, B. G., Telles, S. C. C., Souza, A. C.C., Barros, I. C. S. S., Cortat, J. M., Silva, G. S. \& Ferreira, D. S. (2021b). Esgrima e esgrima em cadeira de rodas. Esporte, Inclusão e Sustentabilidade. Rio de Janeiro: Gama Assessoria Empresarial.

Vigário, P., Pena, B. G., Telles, S. C. C., Souza, A. C.C., Barros, I. C. S. S., Cortat, J. M., \& Jesus, J. S. (2021c). Basquete e basquete em cadeira de rodas. Esporte, Inclusão e Sustentabilidade. Rio de Janeiro: Gama Assessoria Empresarial.

Vigário, P., Pena, B. G., Telles, S. C. C., Souza, A. C.C., Barros, I. C. S. S., Cortat, J. M. (2021d). Vôlei e vôlei sentado. Esporte, Inclusão e Sustentabilidade. Rio de Janeiro: Gama Assessoria Empresarial.

Vitrikas, K., Dalton, H., \& Breish, D. (2020). Cerebral Palsy: An Overview. American Family Physician, 101(4), 213-220.

World Health Organization. (2021). Persons with disabilities tell their stories. Retrieved November 1, 2021, from https://www.who.int/news/item/19-07-2021persons-with-disabilities-tell-their-stories.

Wright, A., Roberts, R., Bowman, G., \& Crettenden, A. (2019). Barriers and facilitators to physical activity participation for children with physical disability: comparing and contrasting the views of children, young people, and their clinicians. Disability and Rehabilitation, 41(13), 1499-1507. https://doi.org/10.1080/09638288.2018.1432702 